\title{
Pregabalin-induced remission in a 62-year-old woman with a 20-year history of vulvodynia
}

\author{
Laurence Jerome MbChB MSc MRCPsych FRCPC
}

L Jerome. Pregabalin-induced remission in a 62-year-old woman with a 20-year history of vulvodynia. Pain Res Manage 2007;12(3):212-214.

A case of a 62-year-old woman presenting with a 20-year history of vulvodynia previously unresponsive to medical treatment is described. The epidemiology, phenomenology and medical management of vulvodynia is reviewed. The case presentation illustrates the role of pregabalin in successful medical management of this chronic pain disorder, as well as the management of common psychiatric morbidities associated with this condition.
Rémission induite par la prégabaline chez une femme de 62 ans souffrant de vulvodynie depuis 20 ans

On décrit ici le cas d'une femme de 62 ans atteinte de vulvodynie depuis 20 ans et jusqu'alors réfractaire aux traitements médicaux. L'épidémiologie, la phénoménologie et le traitement médical de la vulvodynie sont également passés en revue. Ce cas illustre le rôle de la prégabaline dans la prise en charge médicale efficace de ce douloureux problème chronique et le traitement des morbidités psychiatriques courantes associées à cette maladie.

Key Words: Gabapentinoids; Pregabalin; Vulvodynia

$\mathrm{B}$ arbara Reed (1), in her excellent review of the history of the term 'vulvodynia' and current classification, notes that "though vulvodynia was described in 1889 as excessive sensitivity" of the vulva, it rarely was referred to in the medical literature until the 1980s. In 1983, the recognition of this disorder and its effects on the lives of women worldwide led to the adoption of the term vulvodynia by the International Society for the Study of Vulvovaginal Disease. At the time, it was defined as "chronic vulvar discomfort that is characterized by the complaint of burning, stinging, irritation, or rawness" in the absence of skin disease or infection. The International Society for the Study of Vulvovaginal Disease recently revised the definition to include two subgroups: localized and generalized vulvar dysesthesia (2). Each of these subgroups is further categorized as provoked, spontaneous or mixed. It is unclear whether these groups represent separate disorders or different presentations of the same disorder. The term 'vulvar vestibulitis' is no longer used because inflammation is not a prominent component of the disorder; it is now referred to as localized vulvar dysesthesia or vestibulodynia.

Reed comments that the reported prevalence of vulvar pain in different settings is much more common than previously believed, with rates of $15 \%$ in one gynecologist's practice, $1.7 \%$ in an Internet survey and $8.6 \%$ in a population-based study (3) of symptomatic women in Boston, Massachusetts. These findings would extrapolate to more than 2.4 million women in the United States and approximately 15 affected women in a family practice of 2000 patients. Reed notes that the age range is broad, from children (rarely) to women 80 years of age and older, but most women with this disorder are between 20 and 50 years of age (1). No data on the specific characteristics of vulvodynia in older women were found.

The symptoms are thought to be less prevalent in black populations. There is no convincing evidence of infection being the primary pathogenesis. Immunochemistry studies (4) in patients with vulvodynia have shown an altered density of nerve endings and estrogen receptors. There is often overlap with other pain syndromes.

Several reviews have examined the available therapeutic approaches. Pharmacological treatment with tricyclic antidepressants, carbamazepine and local anesthetics, along with biofeedback and occasional surgical intervention, has had variable results. As far as is known, there are no published case control studies of potential causes of vulvodynia or randomized controlled trials of intervention $(5,6)$.

Scheinfeld (7) discusses a group of chronic, neuropathic focal pain syndromes, the 'dynias', that have a predilection for orocervical and urogenital regions and are responsive to gabapentin. They include glossodynia, carotidynia, vulvodynia, orchidynia, prostatodynia, coccygodynia and proctodynia. In some cases the dynias occur secondarily, but more often, despite an exhaustive evaluation, no etiology is found. Two case series $(8,9)$ of vulvodynia have shown good clinical response to gabapentin.

Ben-David and Friedman (8) described 17 patients with a median age of 62 years (range 26 to 82 years) and a mean duration of illness of 4.2 years, not responsive to other treatments. Of these patients, $14(82 \%)$ showed a remission of symptoms after trials of gabapentin after two to four weeks treatment at doses ranging from $900 \mathrm{mg}$ to $1200 \mathrm{mg}$ in divided

The University of Western Ontario, London, Ontario

Correspondence: Dr Laurence Jerome, 90 Wharncliffe Road South, London, Ontario N6J 2K1. Telephone 519-432-3818, fax 519-432-1748, e-mail ljerome@rogers.com 
doses. Benefits were maintained up to 32 weeks. A second, smaller, case series by Bates and Timmins (9) described two women in their 30s. One woman showed an immediate resolution of symptoms, which returned when the medication was discontinued and resolved again on reintroduction of the medication; this effect was maintained over two months at a dose of $900 \mathrm{mg}$ in divided daily doses. The second woman had a remission of symptoms at a dose of $1200 \mathrm{mg}$ in divided doses over three weeks, with benefits maintained at 12 months.

\section{CASE PRESENTATION}

A 62-year-old married woman was referred by her dermatologist for a psychiatric opinion. She came with a diagnosis of vulvodynia. The patient had a 20-year history of symptoms of vulvodynia that had waxed and waned over the years but had become more severe in the past three years with no obvious environmental stressor. Her symptom was not associated with rash. The patient found the burning to be unbearable and experienced significant functional impairment in her activities of daily living. She had difficulty being out of the house for very long and had become largely housebound. There had been a long-standing cessation of sexual activity. Physical examination of the genital area was normal. The patient's symptoms of burning and pain involved the genital area and, at times, included the rectum. The case description conformed to a generalized spontaneous vulvodynia.

There had been a positive marital relationship. The couple had adopted a son who had suffered from attention deficit hyperactivity disorder and learning disabilities but was now an independent adult. The patient had a range of gynecological problems over the years. She had a hysterectomy in 1958 for a growth on her ovary with a later tubal ligation and a cholecystectomy. At the time of the initial assessment, the patient was taking omeprazole for 'a nervous stomach', methyldopa for hypertension and acetaminophen for chronic osteoarthritis. She used a small dose of lorazepam to assist in sleep at night, which along with citalopram reduced the intensity of vulvodynia.

Previous attempted therapies included avoidance of irritants and tight clothing and keeping the area lubricated. Trials of antidepressants including amitriptyline up to doses of $50 \mathrm{mg}$ had shown minimal improvement. Recent trials of citalopram at $20 \mathrm{mg}$ and lorazepam at $1 \mathrm{mg}$ had been moderately helpful. Trials of estrogen cream and vulval pessaries had shown minimal benefit.

At the initial clinical interview, she presented as a pleasant woman who talked about her nervous temperament. She had a fear of vomiting and a fear that she would choke on her own vomit. She had been chronically nervous and suffered from obsessional ruminations focused mainly on her vaginal symptoms throughout much of her adult life. Self-report depression and anxiety scales (Hospital Anxiety and Depression Scale, the Beck Depression Inventory and the Beck Anxiety Inventory) indicated no subjective symptom burden of depression but chronic generalized anxiety symptoms of moderate severity. Initial therapies involved trying to optimize symptom response to citalopram by increasing the dose to $40 \mathrm{mg}$ with adjunctive treatment with amitriptyline at a dose of $10 \mathrm{mg}$. Overall, she believed that her discomfort had improved $30 \%$ to $40 \%$ on the combination of the higher dose of citalopram and amitriptyline. However, she remained an anxious, obsessive individual and continued to be housebound.

Initial treatment with moderate response to anxiety and vaginal pain was discontinued because she developed an independent mitral valve incompetence and cardiac failure requiring a course of digoxin and an anticoagulant. The patient later underwent mitral valve surgery and cataract surgery, which took precedence over her treatment for vulvodynia. Although she was certainly calmer and less anxious and obsessional while on citalopram, her vulvodynial symptoms continued relatively unchanged. She reported a reduction in the intensity of her emotional distress in relation to the vulvodynial symptoms by approximately $40 \%$ to $50 \%$, although the intensity of the pain on a 10-point visual analogue scale had reduced by only $10 \%$ to $20 \%$.

Because of the neuropathic qualities of the vulvodynia, a trial of pregabalin was undertaken at an initial starting dose of $150 \mathrm{mg}$, three times daily, with meals. After 12 weeks, the patient reported an $80 \%$ reduction in pain symptoms, which she attributed to the pregabalin. She had reduced her dose to a single nighttime dose because of some lightheadedness.

She reported that she had been able to resume normal social activities, which she had not been able to do for quite a number of years. For example, she was able to travel on her own to a nearby city to watch her favourite singer in concert, which she not been able to contemplate in the past. She reported "feeling better emotionally than she did for the last 30 years". She was now contemplating applying to re-obtain her driving license because of her increased social mobility.

Her current dose of pregabalin is $250 \mathrm{mg}$ at nighttime and she continues to take citalopram at $20 \mathrm{mg}$. The amitriptyline was discontinued. The current significant benefits in terms of reduced pain intensity for the vulvodynia and significant reduction in anxiety symptoms have continued over a sixmonth period.

\section{DISCUSSION}

The present case adds to the literature of the case series of gabapentinoids producing a remission of symptoms in vulvodynia. The present case is unique in that the duration of symptoms of vulvodynia was in excess of those previously reported and had not remitted spontaneously, which has been reported in the literature (1). However, in other ways, the present case conforms to the clinical case descriptions published in the literature.

The benefits of pregabalin were evident in the remission of functional impairment of pain symptoms, as well as the remission of psychiatric symptoms of obsessional rumination and generalized anxiety that had only previously partially responded to standard treatment with a selective serotonin reuptake inhibitor. It would appear that pregabalin was likely exerting its effect through a dual action - an antinociceptive as well as an antianxiety mechanism.

As far as is known, this is the first report of pregabalin being effective in a case of vulvodynia. Pregabalin effects were delayed in comparison with previous cases of gabapentin treatment, not reaching optimal benefit until approximately 12 weeks. The dose of pregabalin was relatively low compared with previous case reports utilizing gabapentin, but the medication was well tolerated and efficacious at a once daily dose of $250 \mathrm{mg}$. If further studies can replicate these observations, once-daily dosing will likely enhance adherence to treatment. 


\section{REFERENCES}

1. Reed BD. Vulvodynia: Diagnosis and management. Am Fam Physician 2006;73:1231-8.

2. Moyal-Barracco M, Lynch PJ. 2003 ISSVD terminology and classification of vulvodynia: a historical perspective. J Reprod Med 2004:49:772-7

3. Harlow BL, Stewart EG. A population-based assessment of chronic unexplained vulvar pain: Have we underestimated the prevalence of vulvodynia? J Am Med Womens Assoc 2003;58:82-8.

4. Fischer G. Management of vulvar pain. Dermatol Ther 2004;17:134-49.
5. Lotery HE, McClure N, Galask RP. Vulvodynia. Lancet 2004;363:1058-60.

6. Smart OC, MacLean AB. Vulvodynia. Curr Opin Obstet Gynecol 2003; 15:497-500.

7. Scheinfeld N. The role of gabapentin in treating diseases with cutaneous manifestations and pain. Int J Dermatol 2003;42:491-5.

8. Ben-David B, Friedman M. Gabapentin therapy for vulvodynia. Anesth Analg 1999;89:1459-60.

9. Bates CM, Timmins DJ. Vulvodynia - new and more effective approaches to therapy. Int J STD AIDS 2002;13:210-2. 


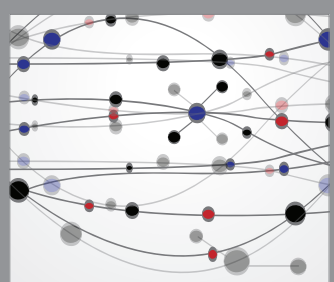

The Scientific World Journal
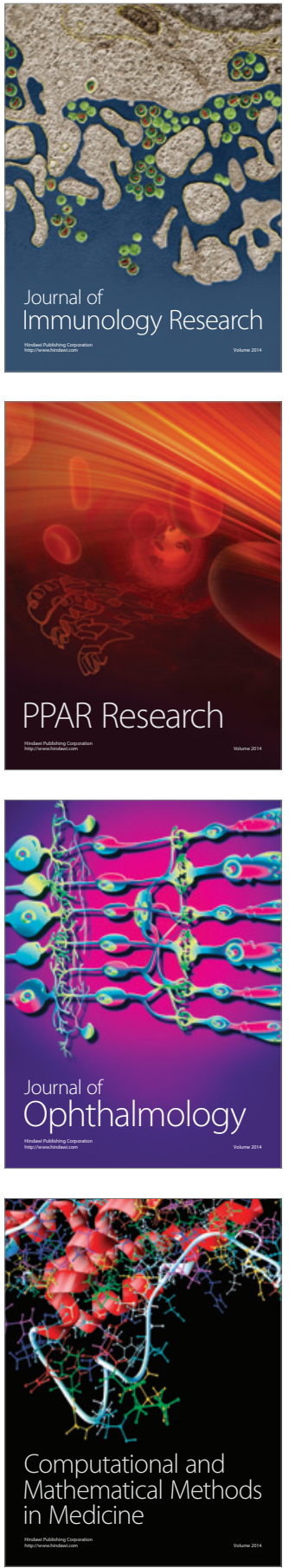

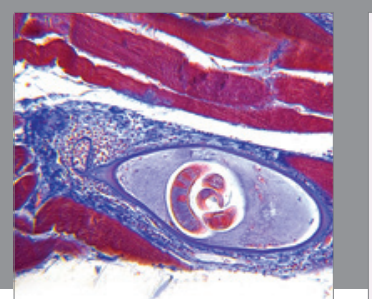

Gastroenterology Research and Practice

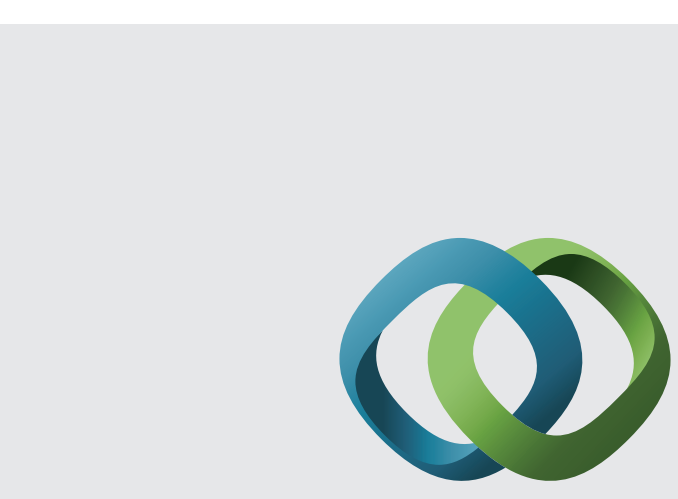

\section{Hindawi}

Submit your manuscripts at

http://www.hindawi.com
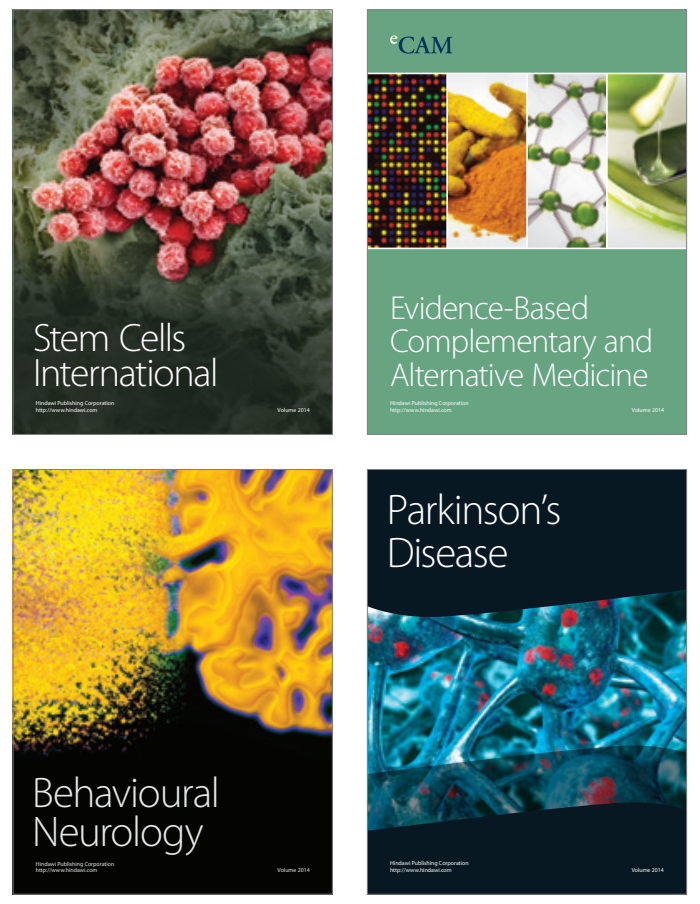
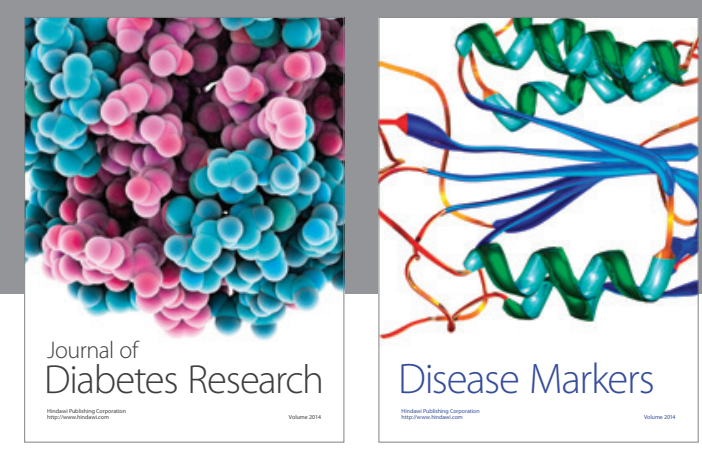

Disease Markers
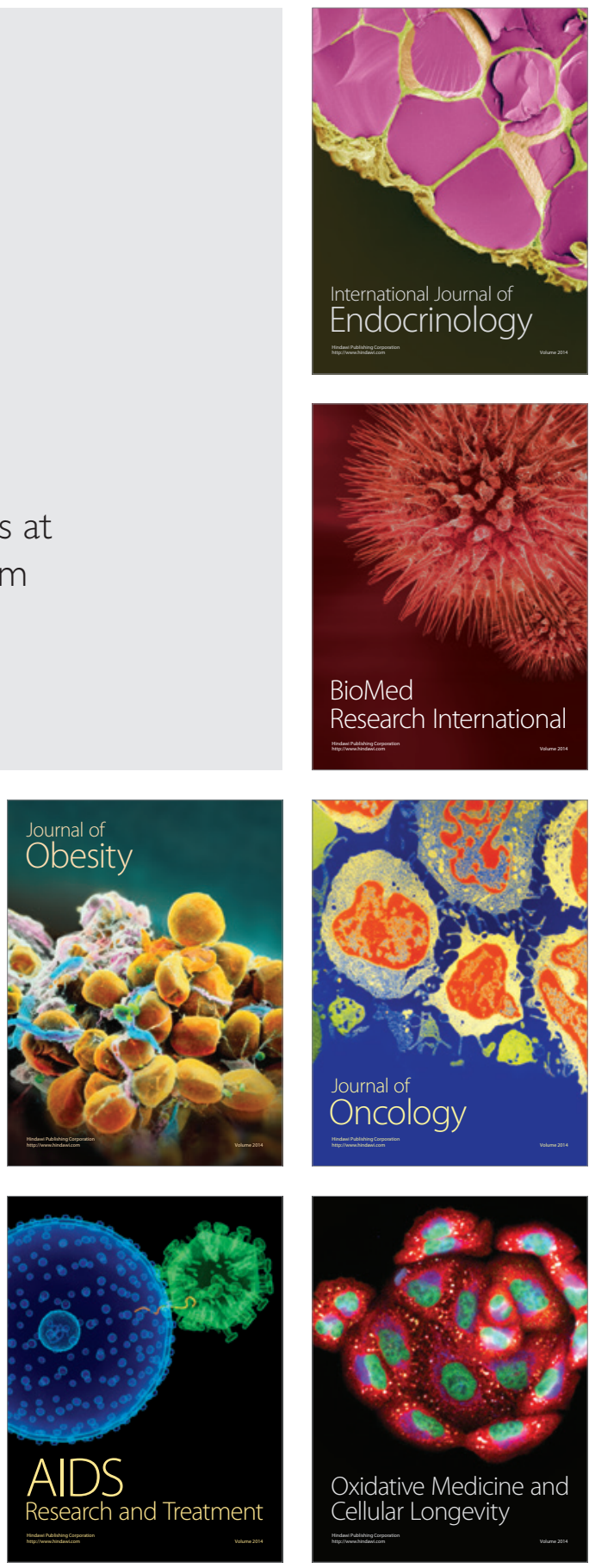